pre-transplant assessment. The median age was $45 \mathrm{yrs}$ (range 26-63), with $41 \%$ female and a median time between scans of 1.4 yrs (range 0.6-3.0). 59 patients (60\%) had two consecutive normal scans. The remaining 40 patients had at least one abnormal scan. $16 \%$ of patients with a normal 1st scan developed an abnormal 2nd scan within a median period of 1.4 years. 28 (70\%) of the patients with an abnormal MPS underwent angiography, of these 12 required revascularisation (either $\mathrm{PCI}$ or $\mathrm{CABG}$ ). Of the remaining 16 patients; 1 died before angiography and the other 15 patients were treated with medical therapy. Of the 59 patients with two normal scans; 3 underwent angiography during the study period (for new symptoms), 1 of these patients required revascularisation after presenting with an ACS. 2 had minor plaque disease.

Conclusions $40 \%$ of SPK patients on the waiting list have an abnormal MPS. Of the patients with normal scans $5 \%$ required an angiogram because of new symptoms with only $2 \%$ requiring revascularisation. Of the patients undergoing angiography driven by MPS 43\% subsequently underwent revascularisation. The current screening interval is successfully monitoring changes in the patients' cardiovascular status with only one patient requiring an intervention which was not predicted by MPS. Therefore a near annual MPS is a useful, non-invasive means by which to monitor patients at very high risk of asymptomatic cardiovascular disease while awaiting a SPK transplant.

\section{VALIDATION OF THE BCIS-1 MYOCARDIAL JEOPARDY SCORE USING CARDIAC MRI}

doi:10.1136/heartjnl-2011-300198.124

${ }^{1} \mathrm{G}$ D J Morton, ${ }^{2} \mathrm{~K}$ De Silva, ${ }^{1} \mathrm{M}$ Ishida, ${ }^{1} \mathrm{~A}$ Chiribiri, ${ }^{1} \mathrm{~A}$ Indermuhle, ${ }^{1} \mathrm{~A}$ Schuster, ${ }^{2} S$ Redwood, 'E Nagel, 'D Perera. 'King's College London, London, UK; 'uy's and St Thomas' NHS Foundation Trust, London, UK

Introduction The recently described angiographic BCIS-1 Myocardial Jeopardy Score (BCIS JS) was designed to classify the extent of coronary artery disease (CAD). It provides a semi quantitative estimate of the amount of myocardium at risk as a result of severe coronary stenoses $(0=$ no jeopardy; $12=$ maximum jeopardy). Advantages include ease of use and universal applicability including classification of left main stem disease and CABG. However anatomic tests, including the BCIS JS, do not incorporate myocardial ischaemia and scar, which are important for management and prognosis. Cardiac magnetic resonance (CMR) imaging allows reliable assessments of myocardial ischaemia and scar in a single examination and was used to examine the functional relevance of the BCIS JS.

Methods 60 consecutive patients with angina and known or suspected $\mathrm{CAD}$ referred for diagnostic x-ray coronary angiography underwent CMR examination at a single UK centre. CMR included standard functional and scar imaging and also high-resolution $\mathrm{k}-\mathrm{t}$ accelerated adenosine stress and rest perfusion imaging at 1.5T (40 patients) or 3T (20 patients). Expert observers blinded to the clinical data analysed the angiographic and CMR data. The BCIS JS was calculated from visual analysis of the coronary angiogram. CMR perfusion and scar data were segmented according to the standard 17-segment model excluding the apex. Segments were subdivided into equal endo- and epicardial sub-segments, each assigned $3 \%$ of the total myocardial volume and classified as normal, ischaemia or scar. Myocardial ischaemia and scar burden were calculated and correlated with the BCIS JS individually and as a combined score (Abstract 124 figure 1).

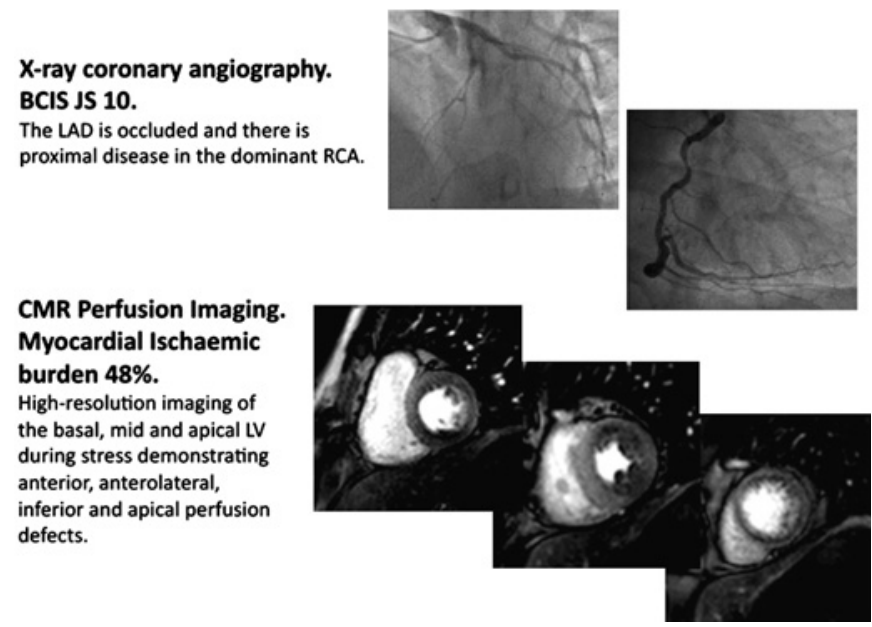

Abstract 124 Figure 1

Results Patient characteristics are summarised in the Abstract 124 table 1.2 patients were excluded (1 claustrophobia; 1 incomplete imaging data). Mean interval \pm SD between CMR and coronary angiography was $40 \pm 47$ days. 13 patients (22\%) with no history of myocardial infarction had CMR evidence of prior infarction. There was a strong correlation between the BCIS JS and myocardial ischaemic burden: Pearson's $r=0.75, p<0.00001$ (Abstract 124 figure 2 ). The BCIS JS was also correlated with the combined burden of scar and ischaemia: $r=0.77, p<0.00001$. There was no difference between 3T and 1.5T CMR imaging. Area under the receiver-operating characteristic curve for BCIS JS to detect $\geq 10 \%$ myocardial ischaemic burden was 0.87 (95\% CI 0.77 to 0.97 ). BCIS JS $\geq 6$ predicted $\geq 10 \%$ myocardial ischaemic burden with sensitivity $68 \%$ and specificity $90 \%$.

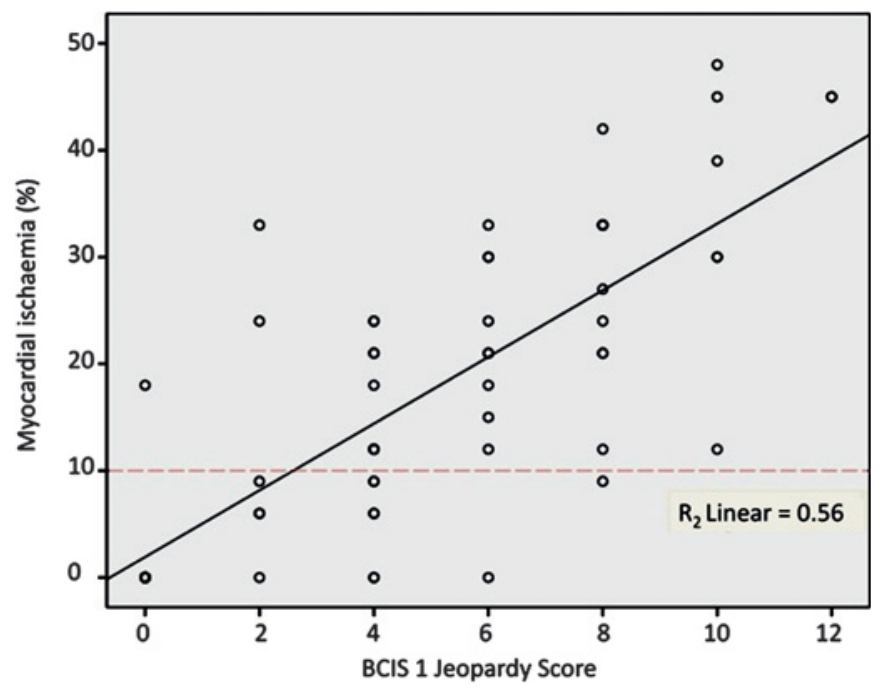

Abstract 124 Figure 2 Correlation between myocardial ischemic burden and BCIS JS.

Conclusions The BCIS JS correlated well with ischaemic burden on CMR. A BCIS JS $\geq 6$ predicts the prognostically important ischaemic threshold of $10 \%$ with high specificity. As expected, the correlation is imperfect which is likely to be a result of difficulty predicting haemodynamic effects of angiographically moderate disease, microvascular disease and limitations of CMR imaging. 
Abstract 124 Table 1

\begin{tabular}{ll}
\hline Characteristic & Number of patients \\
\hline Age (mean \pm SD) & $65 \pm 10$ \\
Left ventricular ejection fraction (mean \pm standard deviation) & $59 \pm 14 \%$ \\
Male & $48(83 \%)$ \\
Diabetes & $17(29 \%)$ \\
Previous CABG & $13(22 \%)$ \\
Previous percutaneous coronary intervention & $22(38 \%)$ \\
Previous MI & $10(17 \%)$ \\
Hypertension & $38(66 \%)$ \\
\hline
\end{tabular}

\section{ASSESSING PATIENT BENEFIT FROM THE REVASCULARISATION OF CHRONICALLY OCCLUDED CORONARY ARTERIES BY ADVANCED CARDIOVASCULAR MRI TECHNIQUES}

doi:10.1136/heartjnl-2011-300198.125

${ }^{1} \mathrm{~N} J$ Artis, ${ }^{2} \mathrm{~A}$ Crean, ${ }^{1} \mathrm{~A}$ Zaman, ${ }^{1} \mathrm{~S}$ Sorbron, ${ }^{1} \mathrm{~A} N \mathrm{~N}$ Mather, ${ }^{1} \mathrm{~S} \mathrm{G}$ Ball, ${ }^{1} \mathrm{~S}$ Plein, ${ }^{1} \mathrm{~J}$ P Greenwood. ' University of Leeds, Leeds, UK; ${ }^{2}$ Toronto General Hospital, Toronto, Canada

Background Cardiovascular magnetic resonance (CMR) imaging can provide an array of information about cardiac function and anatomy. The utility of CMR in the setting of coronary artery chronic total occlusion (CTO) has not been fully investigated. We set out to examine the ability of CMR to show regional improvements in left ventricular (LV) function and perfusion and to investigate if any features were able to predict those that benefit from revascularisation. Methods Twenty-seven patients with single vessel CTO were recruited from clinical waiting lists and underwent a comprehensive CMR assessment prior to and 6 months following attempted CTO revascularisation. A multi-parametric CMR protocol was performed which included cine imaging to assess regional wall thickness/ thickening and global LV function, rest and adenosine stress perfusion imaging (Fermi model), low dose dobutamine stress to assess inotropic reserve, and late gadolinium enhancement (LGE) imaging to determine scar location and extent. Using the AHA 16 segment model only segments supplied by the CTO artery were studied for functional improvement. Data are presented as mean (SD).

Results Procedural success in terms of revascularisation of the occluded artery was achieved in 23 of the 27 patients (85\%, 20 with

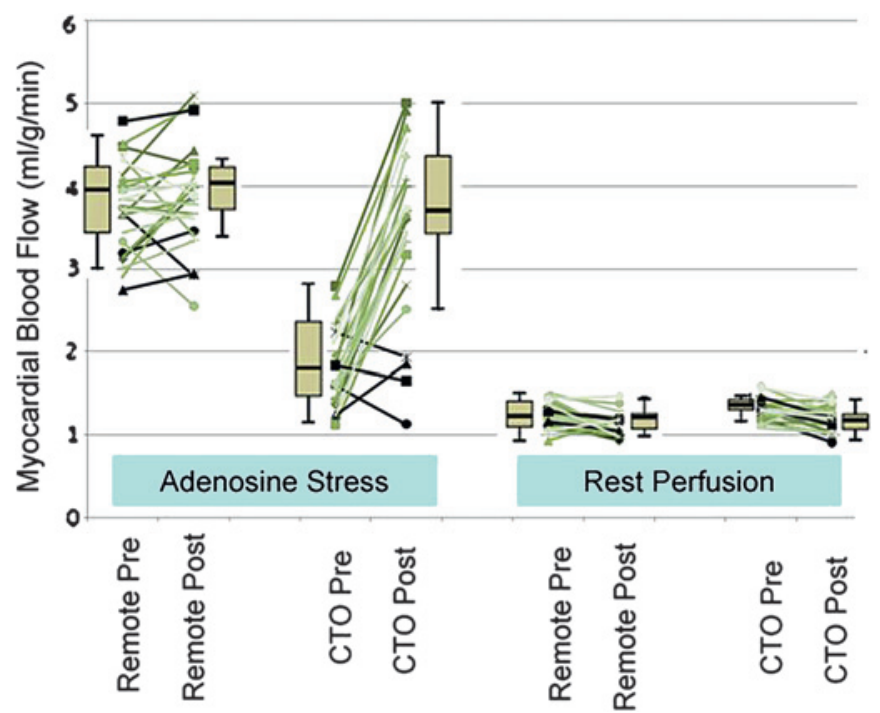

Abstract 125 Figure 1 Changes observed in the CTO and a remote territory following attempted revascularisation. (non-revascularisedblack lines). Adenosine stress increases perfusion only in the CTO territory with no change in resting perfusion.
$\mathrm{PCI}$ and 3 with $\mathrm{CABG}$ ). In those with successful revascularisation by PCI LV volumes reduced (EDV 185 (54) vs 174 (50) p<0.05; ESV $85(60)$ vs $77(58) \mathrm{p}<0.001)$ and the left ventricular ejection fraction improved (56.5(12)\% vs 58.9(12)\% p=0.01). During adenosine stress imaging there was a significant improvement in absolute myocardial blood flow in the revascularised segments (from $1.87(0.51)$ to 3.77 $(0.67) \mathrm{ml} / \mathrm{g} / \mathrm{min} \mathrm{p}<0.001$ ) but not in the remote regions (from 3.76 (0.52) to $3.95(0.58) \mathrm{ml} / \mathrm{g} / \mathrm{min} \mathrm{p}=\mathrm{ns})$. LGE was only present in 25 $(20 \%)$ revascularised segments. In these segments there was a strong inverse correlation between the extent of scar and improvement in segmental systolic thickening $(\mathrm{r}=-0.736, \mathrm{p}<0.001)$. There was a weaker association between the segmental response to low dose dobutamine and the degree of functional improvement following successful revascularisation (Pearson $\mathrm{r}=0.249, \mathrm{p}<0.01$ ).

Conclusion Following revascularisation of $\mathrm{CTO}$, myocardial perfusion increases and both regional and global systolic function improves. While the majority of subjects in this study had no scar on LGE imaging, when segments are scarred there is a negative correlation with improvement in regional systolic thickening.

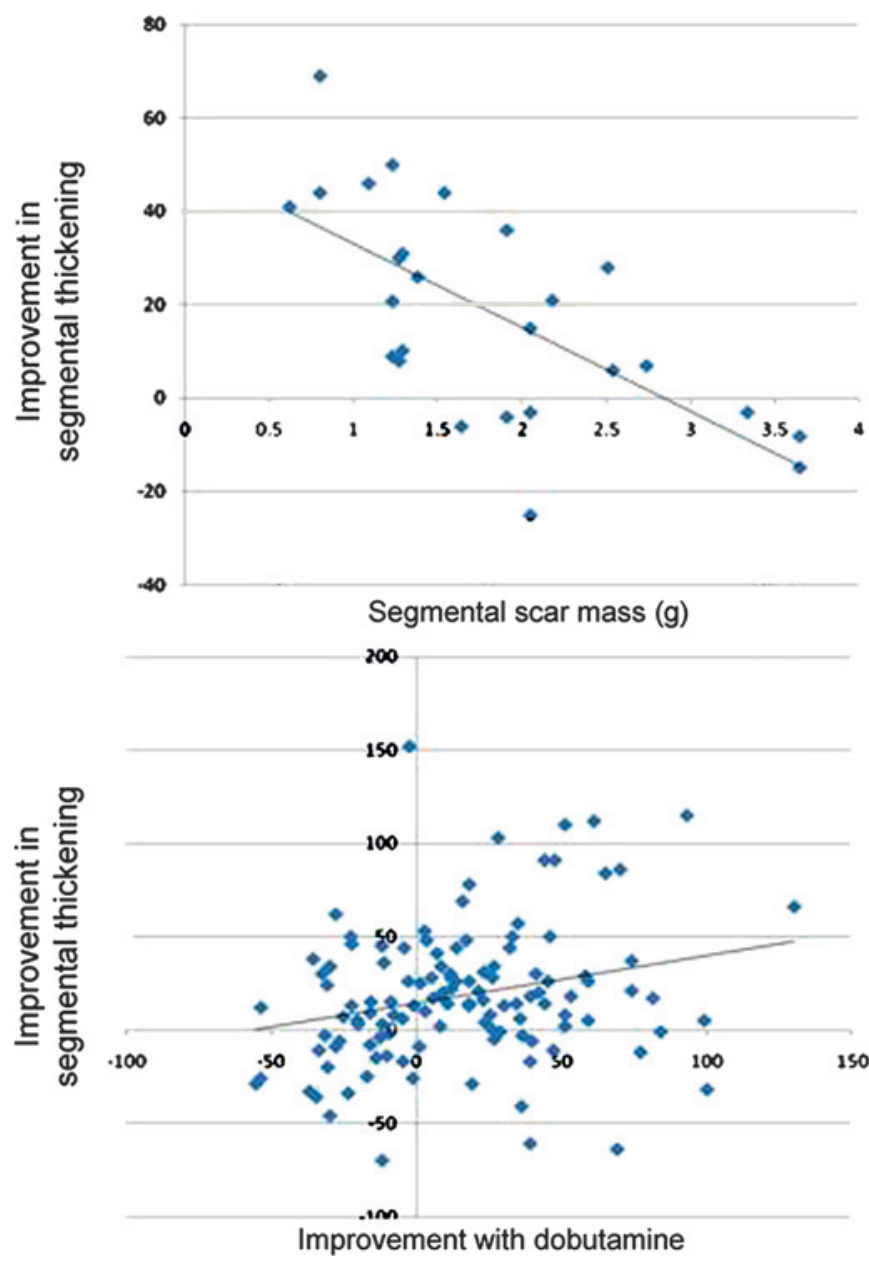

Abstract 125 Figure 2 Relationship of improvement in segmental systolic thickening against segmental scar (top panel) and change in thickening with low dose dobutamine (bottom panel).

\section{THE IMPACT OF NICE GUIDELINES FOR THE INVESTIGATION OF CHEST PAIN ON OUTPATIENT CARDIOLOGY SERVICES IN THE UK}

doi:10.1136/heartjnl-2011-300198.126

${ }^{1} \mathrm{C}$ Patterson, ${ }^{2} \mathrm{E}$ Nicol, ${ }^{3} \mathrm{~L}$ Bryan, ${ }^{4} \mathrm{~T}$ Woodcock, ${ }^{1} \mathrm{~S}$ Padley, ${ }^{1} \mathrm{D}$ Bell. ${ }^{1} / \mathrm{mperial}$ College, London, UK; ${ }^{2}$ Royal Brompton Hospital, London, UK; ${ }^{3}$ Chelsea and Westminster Hospital, London, UK; ${ }^{4}$ NIHR CLAHRC for Northwest London, London, UK 